\title{
Annual direct cost of dry eye in Japan
}

This article was published in the following Dove Press journal:

Clinical Ophthalmology

15 May 2012

Number of times this article has been viewed

\section{Yoshinobu Mizuno \\ Masakazu Yamada \\ Chika Shigeyasu}

Division for Vision Research, National Institute of Sensory Organs, National Tokyo Medical Center, Tokyo, Japan

On behalf of The Dry Eye Survey Group, National Hospital Organization of Japan
Correspondence: Masakazu Yamada Division for Vision Research, National Institute of Sensory Organs, National Tokyo Medical Center, 2-5-I Higashigaoka, Meguro-ku, Tokyo I52-8902, Japan

Tel $+8|334| I$ l III extension $66 \mid 5$

Fax $+8|334||0| 85$

Email yamadamasakazu@kankakuki.go.jp
Background: This study was performed to estimate the annual direct cost incurred by dry eye patients, which includes expenses for treatment and drugs, as well as the cost of punctal plugs.

Methods: The study group consisted of 118 dry eye patients aged 20 years or older who visited any of the 15 medical care facilities that participated in this prospective cohort dry eye study. We estimated annual direct costs from outpatient medical records and survey questionnaires obtained from patients.

Results: Of the total patients enrolled, 10 were men and 108 women, and their average age was $64.1 \pm 11.2$ years. The number of hospital visits made by patients was $5.8 \pm 3.6$ per year. Among those who used ophthalmic solutions, the numbers of bottles used per year were as follows: $32.1 \pm 20.8$ bottles of hyaluronic acid ophthalmic solution (87 patients), $53.1 \pm 42.2$ bottles of artificial tears ( 40 patients), and $33.2 \pm 23.2$ bottles of over-the-counter eyedrops (15 patients). In patients with punctal plugs, $4.1 \pm 3.9$ plugs were used annually. The annual drug cost was $32,000 \pm 21,675$ Japanese yen (323 \pm 219 US dollars). The clinical cost was 16,318 \pm 9961 Japanese yen (165 \pm 101 US dollars). The total direct costs including punctal plug treatment amounted to $52,467 \pm 38,052$ Japanese yen ( $530 \pm 384$ US dollars).

Conclusion: Although treatment modalities for dry eye in Japan were different from those in the US and in European countries, the direct cost of dry eye patients in Japan was comparable with that reported in those countries. Considering the high prevalence of dry eye, the direct cost of this chronic condition may be significant.

Keywords: burden of disease, cost, dry eye, eyedrops, quality of life

\section{Introduction}

Dry eye is recognized as a common eye disease with a high prevalence in many countries, including Japan. ${ }^{1,2}$ Although dry eye rarely leads to blindness or visual impairment, the condition exerts a key influence on quality of life (QOL) and imposes a burden on patients. ${ }^{3-5}$ Miljanovic et al reported that patients with dry eye syndrome have more difficulty in reading, performing professional work, using a computer, watching television, and driving, as compared with those without dry eye. ${ }^{6}$ Utility assessment is a formal method for quantifying the relative impact of a given health state or disease on patient lives, which is defined on a continuous scale from 0 to 1 , where 0 corresponds to the worst possible QOL weight (equal to death) and 1 corresponds to the best possible QOL weight (equal to perfect health). Schiffman et al reported that the mean utility score of moderate dry eye was 0.81 and that of severe dry eye was $0.72 .^{7}$ Hence, there appears to be a considerable burden of dry eye disease based on both its prevalence and patient morbidity. 
In addition to impairment of QOL, the financial burden can be a cause of concern for patients with dry eye. ${ }^{1,8-11}$ Financial burden consists of direct costs, including medical fees and drug expenses, and indirect costs, including absence from work and decreased productivity. Studies to estimate the direct and indirect costs of dry eye have been conducted in European countries and in the US. ${ }^{1,8-11}$ The average annual direct costs of dry eye have been reported to be 600 US dollars in studies from six European countries. ${ }^{8}$ In addition, the indirect financial burden in the form of work absences and decreased productivity cannot be overlooked, with studies showing that patients with dry eye lose $2-5$ working days per year and work with the symptoms for more than 6 months. ${ }^{10,11}$

Assessing multiple aspects of the burden of this disease appears to be important from the perspective of medical care assessment or medical economics. The Dry Eye Survey Group consisting of 15 facilities, mostly affiliated with the National Hospital Organization in Japan, has been conducting a multicenter prospective cohort study on dry eye patients to investigate the effect of the disease from the patient perspective. This article reports the results of analysis on the direct costs of dry eye patients in Japan.

\section{Materials and methods}

This study was performed as part of a multicenter cohort study being conducted at 15 facilities comprising 13 affiliate hospitals of the National Hospital Organization, Keio University, and Tokyo Dental College (see Appendix). The subjects enrolled in the study were dry eye patients aged 20 years and older who visited any of the facilities. The diagnostic criteria used in this study complied with those defined by the Japanese Dry Eye Society, with a slight modification (Table 1). ${ }^{12}$ All cases with definite dry eye according to the criteria were enrolled in the study. The principles of the World Medical Association Declaration of

Table I Diagnosis criteria for dry eye for this study

\begin{tabular}{ll}
\hline Having subjective symptoms due to dry eye & \\
Abnormality in tear function & $<5 \mathrm{~mm}$ \\
I. Schirmer's I testing (without anesthesia) & $<5$ seconds \\
2. Tear film break-up time & \\
$\quad$ Positive when either of I or 2 is applicable & $>3$ \\
Abnormality in corneal and conjunctival epithelium & $>3$ \\
I. Fluorescein staining score (range 0-9) & \\
2. Rose bengal staining score (range $0-9$ ) & \\
Positive when either of I or 2 is applicable
\end{tabular}

Notes: The criteria primarily complied with those defined by Japanese Dry Eye Society with a slight modification. ${ }^{12}$ Definite dry eye was diagnosed when all conditions were met.
Helsinki were followed. Each subject was given a thorough explanation of the purpose of the study and all procedures involved, and the subjects provided written informed consent prior to enrollment. Approval for this research was granted by the Committee for the Protection of Human Subjects at each hospital.

The cases were registered between April 2005 and March 2008 , with a total of 158 cases being initially registered. For the registered cases, information on ocular findings from the responsible physicians was collected. One year after registration in the cohort study, clinical data were collected from each facility. Information on drug expenses and number of hospital visits was also collected from the patients through a survey questionnaire. For this particular report, we analyzed the prescribed drugs and number of hospital visits in one year based on clinical data and patient questionnaire data collected during registration and one year later. Twelve patients were excluded because clinical data at one year after registration could not be obtained from the facilities. Twenty-eight patients were excluded because the survey questionnaire at one year was not returned. Consequently, we used the data from 118 subjects for analysis.

All medical costs are uniformly standardized by the social medical insurance system in Japan. Medical costs associated with dry eye were calculated based on the Japanese Social Insurance Medical Fee Payment for 2008. Based on hospital visits in one year, doctor fees were calculated assuming that an examination, slit-lamp biomicroscopy, and vital staining for corneal and conjunctival epithelium were performed at each hospital visit, and that a Schirmer's test, intraocular pressure measurement, and corrected vision testing were performed once a year. Costs for punctal plugs were calculated from the data based on the number of punctal plugs inserted. Drug costs were the one-year total of the basic preparation charges, charges for issuing prescriptions, and drug costs. The ophthalmic solutions focused on were hyaluronic acid eyedrops, artificial tears, chondroitin sulfate eyedrops, steroid eyedrops, nonsteroidal anti-inflammatory drug eyedrops, and antimicrobial eyedrops and ointments. In the case of patients using over-the-counter eyedrops, numbers of units and expenses were calculated approximately from questionnaires because the details could not be found in outpatient medical records. The direct cost consists of the medical and drug costs, and the expenses of plug insertion.

\section{Results}

The age of the 118 patients ( 10 men, 108 women) enrolled in the study ranged from 33 to 84 years with an average of 
$64.1 \pm 11.2$ years. Of these 118 patients, 47 had Sjögren's syndrome. Accordingly, some patients with Sjögren's syndrome visited the internal medicine department and the ophthalmology department on the same day. There were no ocular comorbidities, such as glaucoma and retinal disorders, which might affect the frequency of hospital visits. Results of clinical tests for dry eye at the time of enrollment and one year later are shown in Table 2. There were no statistically significant differences in the results of clinical tests between enrollment and one year later $(P>0.05$, MannWhitney test).

The annual number of hospital visits made by the 118 patients in the study was $5.8 \pm 3.6$ (range $1-19$ ). With regard to treatment modalities, hyaluronic acid ophthalmic solutions were used by $73.7 \%$ of patients, artificial tears in by $33.9 \%$ of patients, antimicrobials by $15.3 \%$ of patients, steroids by $18.6 \%$ of patients, nonsteroidal anti-inflammatory drugs by $7.6 \%$ of patients, chondroitin sulfate eyedrops by $9.3 \%$ of patients, and over-the-counter eyedrops by $12.7 \%$ of patients (Table 3). Punctal plugs were used by 11 patients $(9.3 \%)$, whereas there were no cases treated with other surgical procedures, such as surgical punctal occlusion or tarsorrhaphy. Among those who used the respective ophthalmic solutions, the numbers of bottles used per year were as follows: $32.1 \pm 20.8$ bottles of hyaluronic acid ophthalmic solution ( 87 patients), $53.1 \pm 42.2$ bottles of artificial tears (40 patients), and $33.2 \pm 23.2$ of over-thecounter eyedrops (15 patients). In patients with punctal plugs, $4.1 \pm 3.9$ plugs were used annually.

From these data, the annual clinical cost was estimated to be $16,318 \pm 9961$ Japanese yen $(165 \pm 101$ US dollars, calculated based on the yen-US dollar exchange rate in March, 2008, Table 4). The pharmacological cost was $32,000 \pm 21,675$ Japanese yen ( $323 \pm 219$ US dollars) per year, and the cost of punctal plugs was $4149 \pm 17,876$ Japanese yen (42 \pm 181 US dollars). The mean annual cost per patient was $52,467 \pm 38,052$ Japanese yen (530 \pm 384 US dollars).

Table 2 Characteristics of patients with dry eye in the survey

\begin{tabular}{lll}
\hline & $\begin{array}{l}\text { At } \\
\text { enrollment }\end{array}$ & $\begin{array}{l}\text { One year } \\
\text { later }\end{array}$ \\
\hline Results of clinical tests (worse eye; $\mathbf{n}=$ I I 8) & \\
Schirmer's testing (mm) & $3.7 \pm 2.8$ & $4.4 \pm 3.5$ \\
Tear film break-up time (sec) & $3.7 \pm 1.7$ & $3.8 \pm 1.6$ \\
Fluorescein staining score (range 0-9) & $1.9 \pm 1.6$ & $2.1 \pm 1.7$ \\
Rose bengal staining score (range 0-9) & $2.0 \pm 2.0$ & $2.0 \pm 1.9$ \\
\hline
\end{tabular}

Notes: Results are expressed as the mean \pm standard deviation. There were no statistically significant differences in results of clinical tests between enrollment and one year later $(P>0.05$, Mann-Whitney test).
Table 3 Treatment modalities for dry eye patients in the survey

\begin{tabular}{lll}
\hline & $\begin{array}{l}\text { Number of } \\
\text { users (\%) }\end{array}$ & $\begin{array}{l}\text { Units used } \\
\text { annually in users }\end{array}$ \\
\hline $\begin{array}{l}\text { Ophthalmic solutions } \\
\text { Hyaluronic acid }\end{array}$ & $87(73.7 \%)$ & $32.1 \pm 20.8$ \\
Artificial tears & $40(33.9 \%)$ & $53.1 \pm 42.2$ \\
Antimicrobial drops & $18(15.3 \%)$ & $13.8 \pm 18.3$ \\
Steroidal drops & $22(18.6 \%)$ & $18.4 \pm 16.5$ \\
NSAID drops & $9(7.6 \%)$ & $13.9 \pm 9.0$ \\
Chondroitin sulfate & II (9.3\%) & $26.3 \pm 17.5$ \\
OTC eyedrops & $15(12.7 \%)$ & $33.2 \pm 23.2$ \\
Punctal plugs & II (9.3\%) & $4.1 \pm 3.9$
\end{tabular}

Note: Numbers of units used for treatment are expressed as the mean \pm standard deviation.

Abbreviations: NSAID, nonsteroidal anti-inflammatory drug; OTC, over-the counter.

\section{Discussion}

In the current study, the annual direct costs for dry eye in Japan were estimated to be 52,467 Japanese yen (530 US dollars), which included clinical costs of 16,318 Japanese yen (165 US dollars), pharmacological costs of 32,000 Japanese yen (323 US dollars), and costs of punctal plugs of 4149 Japanese yen (42 US dollars). It should be noted that treatment modalities for dry eye in Japan are different from those in the US and in European countries. Topical immunosuppressants, such as cyclosporine, and oral medications have not been approved for clinical use in the treatment of dry eye in Japan. Instead, hyaluronic acid eyedrops and artificial tears have been used as the major treatment modalities for dry eye. As shown in Table 3, other treatment modalities, such as steroid eyedrops, over-the-counter eyedrops, and punctual plugs, were concurrently used in some cases. Recently, two new topical agents for treating dry eye, diquafosol tetrasodium $3 \%$ and rebamipide $2 \%$, have been approved for clinical use in Japan. These new drugs may alter the preferred practice patterns for the treatment of dry eye, but were not approved at the time of this study.

Clegg et al reported the results of the annual cost of dry eye patients in six European countries by using statistical data and interviews (Table 5). ${ }^{8}$ Although there was a marked difference between the lowest amount of 273 US dollars in

Table 4 Direct costs for dry eye per year in the survey (Japanese yen)

\begin{tabular}{ll}
\hline Types of costs & Annual costs (mean \pm SD, range) \\
\hline Clinical costs & $16,318 \pm 996 \mid$ (range 2864-53,084) \\
Drug costs & $32,000 \pm 21,675$ (range 48I6-135,944) \\
Costs for punctal plugs & $4149 \pm 17,876$ (range 0-152,320) \\
Mean direct cost per patient & $52,467 \pm 38,052$ (range 7680-294,858) \\
\hline
\end{tabular}

Abbreviation: SD, standard deviation. 
Table 5 Annual costs for dry eye in various countries

\begin{tabular}{|c|c|c|}
\hline Country & Costs (US\$) & Reference \\
\hline France & 273 & Clegg et $\mathrm{al}^{8}$ \\
\hline Germany & 536 & Clegg et $\mathrm{al}^{8}$ \\
\hline Italy & 645 & Clegg et $\mathrm{al}^{8}$ \\
\hline Spain & 765 & Clegg et $\mathrm{al}^{8}$ \\
\hline Sweden & 415 & Clegg et $\mathrm{al}^{8}$ \\
\hline UK & 1100 & Clegg et $\mathrm{al}^{8}$ \\
\hline US & 456 & Pflugfelder' \\
\hline US & $11-355^{*}$ & Enzenauer et $\mathrm{al}^{9}$ \\
\hline US & $221 * *$ & Reddy et al $^{10}$ \\
\hline Japan & 530 & MIZUNO(current study) \\
\hline
\end{tabular}

Notes: *Drug costs only; ${ }^{* *}$ Clinical costs only.

France and the highest amount of 1100 US dollars in the UK, the average expense of 622 US dollars was almost the same as that estimated in our study. The result in the US reported by Pflugfelder of 446 US dollars was also comparable. ${ }^{1}$ Enzenauer et al reported that the annual cost in the particular case of ophthalmic solutions was 11-355 US dollars, which was in the same range as our results, though there is a difference arising from the type of ophthalmic solutions used. ${ }^{9}$ Gayton estimated that 7-10 million Americans spend an average of 320 US dollars per year on artificial tears. ${ }^{13}$ Reddy et al reported that the average doctor fees per year was 211 US dollars. ${ }^{10}$ Although there are differences in preferred treatment modalities for dry eye among countries, there is no marked difference between the costs in the countries compared.

There are possible limitations to our research. In this study, $39 \%$ of patients had dry eye associated with Sjögren's syndrome. The predominance of females (92\%) in the study is partly explained by this comorbidity, although dry eye is usually more common in women than in men. Further, this study was a hospital-based survey rather than a populationbased survey. One of the clinical issues associated with dry eye is that many of the patients have not received medical management. ${ }^{1,2}$ Therefore, it should be noted that the subjects in the study may not be representatives of the majority of patients with dry eye.

There are several studies of other eye diseases that indicate a concern about direct financial burden. It was reported that the direct costs of glaucoma in stage 4 were 2464 US dollars in the US, with approximately half of that amount spent on drugs. ${ }^{14}$ Schmier et al reported that yearly expenditures for latanoprost and travoprost in the US were 1360 US dollars and 1278 US dollars, respectively. ${ }^{15}$ The expenses for diabetic retinopathy are 1118 US dollars in the US, ${ }^{16}$ and for age-related macular degeneration are 7349 Euros in France, 12,445 Euros in Germany, and
5732 Euros in Spain. ${ }^{17}$ Expenditures on other eye diseases are high in comparison with the direct financial burden of dry eye. Drugs for dry eye are comparatively inexpensive and surgical remedies are generally not undertaken except for punctal plugs. However, when considering the high prevalence of this condition, the direct cost of this chronic condition may be significant.

Although not examined in this study, the indirect financial burden of dry eye is equally important, with $7 \%$ of patients obliged to change jobs and $11 \%$ forced to cut back on their working hours. ${ }^{10}$ The subjective symptoms inherent in the disease, including eye discomfort for more than 6 months, contribute to the necessity of taking 2-5 days off from work in a year. In the present study, we have shown that patients with dry eye visit the hospital on average 5.8 times a year. When this time is converted into opportunity cost, it turns out to be approximately 500 US dollars. Although there are differences in indirect financial burden due to economic conditions, working conditions, and calculation methods, depending on each country, this cost is a cause of concern for the patients and cannot be overlooked. The estimation of indirect financial burden of dry eye should be investigated in the future.

The research method used here does not involve an assessment of outcomes and is called cost analysis. Moreover, other methods include cost utility analysis using utility and quality-adjusted life years, and cost effectiveness analysis based on specific outcomes (eg, life years and treatment results) and costs. Although cost analysis is easy to implement, it has a disadvantage in that it cannot be used to determine directly the distribution of medical resources and relative effectiveness of a given strategy for a specific treatment. Therefore, the burden of disease and treatment assessments using cost effectiveness analysis and cost utility analysis are topics for future investigation.

\section{Acknowledgment}

This study was supported by a grant from the National Hospital Organization in Japan.

\section{Disclosure}

The authors report no conflicts of interest in this work.

\section{References}

1. Pflugfelder SC. Prevalence, burden, and pharmacoeconomics of dry eye disease. Am J Manag Care. 2008;14(3 Suppl):S102-S106.

2. Yamada M. Dry eye syndrome: concept, pathogenesis, and therapeutic modalities based on the new definition. J Jpn Ophthalmol Soc. 2008; 113:541-552. 
3. Schiffman RM, Christianson MD, Jacobsen G, Hirsch JD, Reis BL. Reliability and validity of the Ocular Surface Disease Index. Arch Ophthalmol. 2000;118:615-621.

4. Mertzanis P, Abetz L, Rajagopalan K, et al. The relative burden of dry eye in patients' lives: comparisons to a US normative sample. Invest Ophthalmol Vis Sci. 2005;46:46-50.

5. Mizuno Y, Yamada M, Miyake Y. Association between clinical diagnostic tests and health-related quality of life surveys in patients with dry eye syndrome. Jpn J Ophthalmol. 2010;54:259-265.

6. Miljanovic B, Dana R, Sullivan DA, Schaumberg DA. Impact of dry eye syndrome on vision-related quality of life. Am J Ophthalmol. 2007;143:409-415.

7. Schiffman RM, Walt JG, Jacobsen G, Doyle JJ, Lebovics G, Sumner W. Utility assessment among patients with dry eye disease. Ophthalmology. 2003;110:1412-1419.

8. Clegg JP, Guest JF, Lehman A, Smith AF. The annual cost of dry eye syndrome in France, Germany, Italy, Spain, Sweden and the United Kingdom among patients managed by ophthalmologists. Ophthalmic Epidemiol. 2006;13:263-274.

9. Enzenauer RW, Kao A, Williams T, Lambert RW. Relative costs of various preserved artificial tear solutions for the treatment of dry eye conditions. Eye Contact Lens. 2003;29:238-240.
10. Reddy P, Grad O, Rajagopalan K. The economic burden of dry eye a conceptual framework and preliminary assessment. Cornea. 2004;23:751-761.

11. Patel VD, Watanabe JH, Strauss JA, Dubey AT. Work productivity loss in patients with dry eye disease: an online survey. Curr Med Res Opin. 2011;27:1041-1048.

12. Shimazaki J, Tsubota K, Kinoshita S, et al. Definition and diagnosis of dry eye 2006. Atarashii Ganka. 2007;24:181-184. Japanese.

13. Gayton JL. Etiology, prevalence, and treatment of dry eye disease. Clin Ophthalmol. 2009;3:405-412.

14. Lee PP, Walt JG, Doyle JJ, et al. A multicenter, retrospective pilot study of resource use and costs associated with severity of disease in glaucoma. Arch Ophthalmol. 2006;124:12-19.

15. Schmier JK, Covert DW, Robin AL. First-year treatment costs among new initiators of topical prostaglandin analogs. Clin Ophthalmol. 2009;3:637-644.

16. Javitt JC, Canner JK, Sommer A. Cost effectiveness of current approaches to the control of retinopathy in type I diabetics. Ophthalmology. 1989;96:255-264.

17. Cruess AF, Zlateva G, Xu X, et al. Economic burden of bilateral neovascular age-related macular degeneration: multi-country observational study. Pharmacoeconomics. 2008;26:57-73. 


\section{Appendix}

\section{The Dry Eye Survey Group of National Hospital Organization in Japan}

The following individuals and National Hospital Organizations participated in the study:

H Negishi, A Hayashi, Chiba Medical Center, Chiba; Terada H Tachikawa, Disaster Medical Center; T Katsuta, K Fujiike, S Hatou, Tokyo Medical Center, Tokyo; Y Yamada, Tokyo Hospital, Kiyose; H Hirose, K Toura, Nagoya Medical Center, Nagoya; M Yamamoto, N Yoshida, N Kawagoe, Kyoto Medical Center, Kyoto; Y Otori, Y Saito, Y Sakamoto, Osaka National Hospital, Osaka; T Nakamura, Kure Medical
Center and Chugoku Cancer Center, Kure; M Kogiso, Zentsuji National Hospital, Zentsuji; H Enaida, T Nagatomi, Kyusyu Medical Center, Fukuoka; A Takehara, S Kubota, E Niiro, Ureshino Medical Center, Ureshino; H Aoki, Kumamoto Medical Center, Kumamoto; N Miyamura, H Hayashida, Nagasaki Medical Center, Ohmura; M Kaido, M Dogru, K Tsubota, Keio University School of Medicine, Tokyo; S Den, J Shimazaki, Tokyo Dental College, Chiba; M Yamada, Y Mizuno, G Hanazono, K Tsunoda, Y Miyake, National Institute of Sensory Organs, Tokyo Medical Center, Tokyo.
Clinical Ophthalmology

\section{Publish your work in this journal}

Clinical Ophthalmology is an international, peer-reviewed journal covering all subspecialties within ophthalmology. Key topics include: Optometry; Visual science; Pharmacology and drug therapy in eye diseases; Basic Sciences; Primary and Secondary eye care; Patient Safety and Quality of Care Improvements. This journal is indexed on

\section{Dovepress}

PubMed Central and CAS, and is the official journal of The Society of Clinical Ophthalmology (SCO). The manuscript management system is completely online and includes a very quick and fair peer-review system, which is all easy to use. Visit http://www.dovepress.com/ testimonials.php to read real quotes from published authors. 